\title{
Reference Islands Definition within INSULAE Project through KPIs
}

\author{
Laura Giménez De Urtasun1, David M. Rivas-Ascaso, Noemi Galán Hernández¹, \\ Giorgos Papadoupoulos ${ }^{2}$, Kostas Tsatsakis ${ }^{2}$ \\ ${ }^{1}$ CIRCE Foundation, Zaragoza, Spain \\ ${ }^{2}$ Suite5: Data Intelligence Solutions Limited, Gerakas, Greece \\ Email: lgimenez@fcirce.es,dmrivas@fcirce.es,ngalan@fcirce.es, giorgos@suite5.eu, kostas@suite5.eu
}

How to cite this paper: Giménez De Urtasun, L., Rivas-Ascaso, D.M., Hernández, N.G., Papadoupoulos, G. and Tsatsakis, K. (2020) Reference Islands Definition within INSULAE Project through KPIs. Smart Grid and Renewable Energy, 11, 29-49. https://doi.org/10.4236/sgre.2020.113003

Received: March 15, 2020

Accepted: March 27, 2020

Published: March 30, 2020

Copyright $\odot 2020$ by author(s) and Scientific Research Publishing Inc. This work is licensed under the Creative Commons Attribution International License (CC BY 4.0).

http://creativecommons.org/licenses/by/4.0/

\begin{abstract}
Most of the isolated electrical systems throughout the world suffer from similar problems of fragility and high dependence on external resources to generate energy. Smart Grid solutions and integration of renewable energies in order to solve their problems have increased, although it is necessary to know their specific characteristics to select the optimal solutions for each case. Therefore, as the overall objective of INSULAE Project, the development of an Investment Planning Tool, IPT, is on the way. This paper provides a view on a characterization methodology developed for the set of Reference Islands and how it will help to exploit the IPT developed. For that, characterization vectors have been defined based on a selection of Key Performance Indicators (KPIs). And Reference Islands have been obtained from the analysis of KPIs data gathered from EU islands considering the vectors formed. The linkage of new islands to reference islands helps provide the new islands with an assessment on the possibility space of their investment plans with the aim of being a decarbonization plan considering the demonstrations already evaluated.
\end{abstract}

\section{Keywords}

Smart Grid, Islands, KPIs, Reference Typologies

\section{Introduction}

INSULAE project, "Maximising the impact of Innovative energy approaches in the EU Islands" aims to help islands find locally produced, sustainable and low-cost sources of energy. This objective is linked directly to EU policies for decarbonising the energy systems of geographical islands [1]; and with the Sustainable Development Goals [2], particularly with objective 7: Affordable and clean energy, and 
objective 9: Industry, Innovation and infrastructure. The project develops interventions linked to seven replicable use cases at three Lighthouse Islands (in Croatia, Denmark and Portugal). The goal is to demonstrate their capability to develop RES-based systems up to 70\% cheaper than diesel. To assist Europe's policymakers, the project will design an investment planning tool, IPT, to be deployed at four Follower Islands in Germany, Greece, Spain and France DOM/TOM for the improvement of related action plans. In order to guide new users of the IPT, and allow exploitation of its results at any EU island, the project has developed a characterization methodology for any new island, through characterization vectors, and Reference Islands with which any new island should be matched in order to obtain an assessment on the possibilities of its investment plan with the aim to bring in a decarbonization plan.

A first step for any improvement methodology is the establishment and measurement of key performance indicators, KPIs [3]. This needs to be carefully developed to consider the technical achievements and their relationship with the main objectives. Therefore the close connection of INSULAE project with previous initiatives was studied: at Croatian islands [4], with SMILE project [5] and at a general EU level [6]. The work of parametrization was then grouped into the eleven characteristic categories considered relevant. These eleven categories are: electricity generation, electricity demand, electricity networks, physical geography, social, environmental, economic, hydrology, transport, telecommunication, science and technology. A reduced set of KPIs was then identified and used to calculate six vectors: Generation, Demand, Network, Resource Capacity, Society and Environmental. Vectors have been composed using the set of KPIs identified considering weighting factors to indicate the importance of the different KPIs in the objectives targeted by the project.

In order to obtain a representative view on the possible parameters, KPIs, and vectors to consider, and the values of the EU islands, a wide search on the different parameters values in EU islands was performed by gathering information from public sources. In this way, when the method or possible decision on the composition of the vectors had different possibilities, the selection could be made based on the availability of the data across EU islands. Therefore, each vector composition has been designed considering INSULAE objectives as well as availability of data.

With the vectors evaluated in a large set of EU islands, the construction of the reference islands has been developed through an algorithm based on the $k$-means clustering technique [7], obtaining seven reference islands that act as a representative view of the EU islands. After this, INSULAE islands, lighthouse and follower islands, have been linked to one reference island in order to provide the project experiences linked to a reference island to any new island using the IPT.

The following document describes the study developed as follows: Section 2 presents the Establishment of Characterization Vectors; Section 3 describes the process of Data Gathering along EU islands; Section 4 presents the process followed to obtain the Reference islands. Finally, Section 5 offers some discussion on the results and the conclusions gathered during the study. 


\section{Establishment of Characterization Vectors}

The first task deals with the selection of a reduced set of KPIs, based on the parameters obtained in a previous step. For this task it was important to consider previous work done in projects like SMILE, in which KPIs in islands were previously evaluated, and furthermore KPIs currently used by Artelys Crystal software.

From all the sources it was considered important to cover the characterization in islands without losing sight of the main objective of the project, that is the decarbonization of the islands, and the objective of the establishment of a tool that could enable the planning of investments in future islands considering similarities with lighthouse and follower islands and how their demonstrations could help these future islands.

Another important consideration that was used in the selection of the KPIs was the availability of standardized data from public sources. This point has been critical due to the need for gathering information from as many EU islands as possible.

\subsection{Selection of Final Reduced Set of KPIs}

As explained, six vectors were finally chosen from the categorization performed in the first steps and their final set of KPIs selected. In both cases the definition was based on the completion of INSULAE objectives and the data availability that was necessary for the EU stock of islands data gathering, so that afterwards, the definition of Reference Islands was assured.

In all cases, vectors have been designed to provide a value between 0 and 100 .

The six vectors and their set of KPIs are explained below.

\section{Generation Vector}

The aim of this vector is to provide information on the type of generation sources used to provide electricity to an island, and how they contribute to a decarbonization of the island.

Therefore, the reduced set of KPIs selected for the vector were:

Renewable dispatchable generation: providing a view of the amount of electricity consumed in the island that comes from a renewable dispatchable source. Therefore, its value is a result of the sum of the annual generation from sources like hydroelectric, geothermal, bioenergy, combined cycle generators. This sum is later divided by the total island annual electricity demand and multiplied by 100 in order to obtain the percentage.

Renewable non-dispatchable generation: providing a view of the amount of electricity consumed in the island that comes from a renewable non-dispatchable source. Therefore, its value is a result of the sum of the annual generation from sources like wind or solar generators. This sum is afterwards divided by the total island annual electricity demand and multiplied by 100 in order to obtain the percentage.

Interconnection: providing a view of the amount of electricity consumed on 
the island that comes through an interconnection and is not produced on the island. The annual amount of electricity provided through the interconnection is divided by the total island annual electricity demand and multiplied by 100 in order to obtain the percentage.

Conventional generation: providing a view of the amount of electricity consumed on the island that comes from a conventional source on the island. Its value can be obtained using the first three KPIs as:

100 - Renewable dispat. gen. - Renewable non dispat. gen. - Interconnection (1)

\section{Demand Vector}

The aim of this vector is to provide information on the energy demand of the island. Considering both electrical and thermal (fuel) consumption. For this, three main aspects were found to be most representative: Electrical demand, vehicle consumption, and island use of heating or air conditioning, AC, devices. Therefore, the reduced set of KPIs selected for the vector were:

Electricity demand: providing a view of the amount of electricity consumed on the island per capita. Therefore, its value is a result of the island annual demand divided by the island population. In order to obtain a percentage, this value is afterwards standardized by multiplying by 100 and dividing by the maximum value obtained for the whole EU stock of islands considered.

Vehicle demand: providing a view of the amount of energy consumed on the island by vehicles. The value of this KPI is obtained from the number of vehicles per capita on the island and considers an average use of $6.500 \mathrm{~km}$ per year for each vehicle, with an average consumption of 9 litres per $100 \mathrm{~km}$ and an equivalence of $10 \mathrm{kWh}$ for each litre of fuel. In order to obtain a percentage, this value is afterwards standardized by multiplying by 100 and dividing by the maximum value obtained for the whole EU stock of islands considered.

Heating and $\mathrm{AC}$ demand: providing a view of the amount of energy consumed on the island coming from heating and AC devices. This KPI is obtained through the sum of the Heating Degree Days, $\mathrm{HDD}^{1}$ and Cooling Degree Days, $\mathrm{CDD}^{2}$, which is afterwards multiplied by the average energy consumption for heating or cooling per degree day and inhabitant (2.4 kWh/DD and inhabitant). In order to obtain a percentage, this value is afterwards standardized by multiplying by 100 and dividing by the maximum value obtained for the whole EU stock of islands considered.

\section{Network Vector}

The aim of this vector is to provide information on the quality of the electricity consumed by the island. This factor directly affects the quality of life and the probability of companies being based on the island. The reduced set of KPIs selected for the vector were:

${ }^{1} \mathrm{~A}$ heating degree day (HDD) is a measurement designed to quantify the demand for energy needed to heat a building. It is the number of degrees that a day's average temperature is below $18^{\circ}$ Celsius, which is the temperature below which buildings need to be heated.

${ }^{2} \mathrm{~A}$ cooling degree day (CDD) is a measurement designed to quantify the demand for energy needed to cool buildings. It is the number of degrees that a day's average temperature is above $18^{\circ}$ Celsius. 
Network use factor: providing a view of the ratio of use of the whole capacity of the network. Therefore, its value is a result of the annual electricity demand of the island divided by the electricity use of the island in the hypothetical case that the electricity peak demand was maintained for the whole year. In order to obtain a percentage, this value is afterwards standardized by multiplying by 100 and dividing by the maximum value obtained for the whole EU stock of islands considered.

Interconnection capacity: providing a view of the amount of electricity that can be provided from the interconnections of the island in reference to the electricity peak demand. The value of this KPI is obtained from dividing the interconnection capacity by the peak annual demand. In order to obtain a percentage, this value is afterwards standardized by multiplying by 100 and dividing by the maximum value obtained for the whole EU stock of islands considered.

Storage capacity: providing a view of the amount of energy that could be managed through storage, considering storage facilities on the island (batteries, pumped hydro or other storage technologies) in reference to the electricity demand peak. The value of this KPI is obtained by dividing the storage capacity by the peak annual demand. In order to obtain a percentage, this value is afterwards standardized by multiplying by 100 and dividing by the maximum value obtained for the whole EU stock of islands considered.

SAIDI $^{3}$ equivalent: providing a view of the electricity reliability on the island. The island value is subtracted from the maximum value obtained for the EU stock of islands considered, so that a low average duration of interruptions would increase the value of the KPI. In order to obtain a percentage, this value is afterwards standardized by multiplying by 100 and dividing by the maximum value obtained for the whole EU stock of islands considered.

\section{Resource capacity Vector}

The aim of this vector is to provide information on the possibilities of the island to host more renewable energy than the actual installed capacity and therefore the possibilities to improve their decarbonization. The reduced set of KPIs selected for the vector were:

Surface to cover with solar: providing a view of the use of the islands surface that would be necessary to increase solar generation so that it could cover $100 \%$ of non-renewable covered demand. This estimate considers the global horizontal irradiation for the island. Therefore, its value comes from the island annual demand not covered by renewable sources, and the surface that new solar generators covering that demand would use. The value is given as a percentage of the islands' area. In order to obtain a KPI with values from 0 to 100 , this value is afterwards standardized by multiplying by 100 and dividing by the maximum value obtained for the whole EU stock of islands considered.

Surface to cover with wind farms: providing a view of the use of the islands surface that would be necessary to increase wind generation so that it could cover ${ }^{3}$ System Average Interruption Duration Index - a system index of average duration of interruption in the power supply indicated in minutes per customer. 
$100 \%$ of non-renewable covered demand. This estimate considers the wind speed $(V)$ as $10 \%$ of the value at the windiest location on the island. More considerations have been made:

- For average atmospheric conditions of density and moisture constant: Power per sq. meter $=0.0006 \mathrm{~V}^{\beta}$;

- An average turbine, with a capacity of 2.5 - $3 \mathrm{MW}$, would have a rotor of $D=$ $120 \mathrm{~m}$, which would cover a wind surface of: $S=\pi(D / 2)^{2}=11.30973 \mathrm{~m}^{2}$;

- Land area for each wind turbine: $L=10 D \times 5 D=6.000 \mathrm{~m}^{2}$;

- Wind turbine efficiency $35 \%$.

Therefore, its value comes from the island annual demand not covered by renewable sources, and the surface that new wind generators covering that demand would use. The value is given as a percentage of the islands' area. In order to obtain a KPI with values from 0 to 100 , this value is afterwards standardized by multiplying by 100 and dividing by the maximum value obtained for the whole EU stock of islands considered.

\section{Society Vector}

The aim of this vector is to provide information on the quality of life on islands. Considering that it can be affected directly through an investment plan, the reduced set of KPIs selected were:

Population density: providing a view of the amount of resources needed for the population on the island. Its value is a result of the island population divided by the island area. In order to obtain a percentage, this value is afterwards standardized using the Average EU population density, a value of 121.08 inhabitants per square kilometer.

Regional GDP: providing a view on the differences in the cost of living and inflation rates on the stock of islands considered. The value of this KPI is obtained directly from one source [8]. In order to obtain a percentage, this value is afterwards standardized by multiplying by 100 and dividing by the maximum value obtained for the whole EU stock of islands considered.

Unemployment rate: providing a view of the amount of unemployed people on the island, and the economy of the island. The island value is subtracted from the maximum value obtained for the EU stock of islands considered, so that a low unemployment rate would increase the value of the KPI. In order to obtain a KPI with values from 0 to 100 , this value is afterwards standardized by multiplying by 100 and dividing by the maximum value obtained for the whole EU stock of islands considered.

\section{Environmental Vector}

The aim of this vector is to provide information on the needs of decarbonization actions on the island. Considering that this factor can be affected directly through an investment plan, the reduced set of KPIs selected for the vector were:

Air pollution: providing a view of the amount of pollutant particles in the air and therefore of the air quality. Its value is given on a scale of 1 to 5.1 being the value that corresponds to less than $5 \mu \mathrm{g} / \mathrm{m}^{3}$, and 5 the value that corresponds to 
more than $25 \mu \mathrm{g} / \mathrm{m}^{3}$. The island value is subtracted from the maximum value obtained for the EU stock of islands considered, so that low air pollution would increase the value of the KPI. In order to obtain a percentage, this value is afterwards standardized by multiplying by 100 and dividing by the maximum value obtained for the whole EU stock of islands considered.

GHG emissions: providing a view of the amount of Green House Gas emissions emitted by the island and therefore of the air quality. Its value is given on a scale of 1 to 5.1 being the value that corresponds to less than 4 tonnes per capita, and 5 the value that corresponds to more than 13 tonnes per capita. The island value is subtracted from the maximum value obtained for the EU stock of islands considered, so that low GHG emissions would increase the value of the KPI. In order to obtain a percentage, this value is afterwards standardized by multiplying by 100 and dividing by the maximum value obtained for the whole EU stock of islands considered.

Water risk: providing a view of the ratio of total water withdrawals to available renewable surface and groundwater supplies. Its value is given on a scale of 1 to 5.1 being the value that corresponds to less than $10 \%$, and 5 the value that corresponds to more than $80 \%$. The island value is subtracted from the maximum value obtained for the EU stock of islands considered, so that low water risk would increase the value of the KPI. In order to obtain a percentage, this value is afterwards standardized by multiplying by 100 and dividing by the maximum value obtained for the whole EU stock of islands considered.

\subsection{Characterization Vectors Composition}

With the reduced set of KPIs selected and defined the next step is the composition of the characterization vectors. For each vector KPIs have already been proposed, although it is still necessary to provide a weighting factor to determine the way each KPI influences the characterization vector. Each characterization vector will be valued between 0 and 100. As the KPIs have been designed the same way, it means that weighting factors will sum to 1 for each characterization vector; as shown in the following generic equation.

$$
\text { Vector } i=a * \mathrm{KPI}_{i a}+b * \mathrm{KPI}_{i b}+c * \mathrm{KPI}_{i c} \text { where } a+b+c=1
$$

The weighting factors chosen for each characterization vector considering Insulae project objectives are shown below:

\section{Generation Vector}

As previously explained, the aim of this vector is to provide information on the type of generation sources used to provide electricity to an island, and how they contribute to decarbonization of the island. The four KPIs selected were:

- Renewable dispatchable generation (Ren.Disp.Gen.);

- Renewable non-dispatchable generation (Ren.NonDisp.Gen.);

- Interconnection (Intercon.);

- Conventional generation (Convent.Gen.). 
The chosen weighting factors are:

$$
\begin{aligned}
\text { Generation }= & 0.25 * \text { Ren.Disp.Gen. }+0.15 * \text { Ren.NonDisp.Gen } \\
& +0.1 * \text { Intercon. }+0.5 *(100-\text { Convent.Gen. })
\end{aligned}
$$

\section{Demand Vector}

As previously explained, the aim of this vector is to provide information on the amount of energy demanded by the island. Considering both electrical and thermal (fuel) consumption. For this, three KPIs were selected:

- Electric demand (Elec.Dem.);

- Vehicles demand (Veh.Dem.);

- Heating and AC demand (Heat \& AC.Dem.).

The chosen weighting factors are:

Demand $=0.34 *$ Elect.Dem. $+0.33 *$ Veh.Dem. $+0.33 *$ Heat \& AC.Dem.

\section{Network Vector}

As previously explained, the aim of this vector is to provide information on the quality of the electricity consumed by the island. For this, four KPIs were selected:

- Network use factor (Net.Use);

- Interconnection capacity (Interc.Cap.);

- Storage capacity (Storag.Cap.);

- SAIDI equivalent (SAIDI).

The chosen weighting factors were:

Network $=0.1 *$ Net.Use $+0.2 *$ Interc.Cap. $+0.2 *$ Storag.Cap. $+0.5 *$ SAIDI

\section{Resource capacity Vector}

As previously explained, the aim of this vector is to provide information on the possibilities on the island to host more renewable energy than the actual installed capacity and therefore the possibilities to improve their decarbonization. For this, two KPIs were selected:

- Surface to cover with solar (Surf.Solar);

- Surface to cover with wind farms (Surf.Wind).

The chosen weighting factors were:

$$
\text { Resource Capacity }=0.5 * \text { Surf.Solar }+0.5 * \text { Surf.Wind }
$$

\section{Society Vector}

As previously explained, the aim of this vector is to provide information on the quality of life on islands. For this, three KPIs were selected:

- Population density (Popul.Dens.);

- Regional GDP (Region.GDP);

- Unemployment rate (Unemploy).

The chosen weighting factors were:

$$
\text { Society }=0.3 * \text { Popul.Dens. }+0.35 * \text { Region.GDP }+0.35 * \text { Unemploy }
$$




\section{Environmental Vector}

As previously explained, the aim of this vector is to provide information on the need for decarbonization actions on the island. For this, three KPIs were selected:

- Air pollution (Air.Pollut.);

- GHG emissions (GHG.Emission);

- Water risk (Water.Risk).

The chosen weighting factors were:

$$
\begin{aligned}
\text { Environmental }= & 0.33 * \text { Air.Pollut. }+0.33 * \text { GHG.Emission } \\
& +0.34 * \text { Water.Risk }
\end{aligned}
$$

\subsection{Characterization Vectors and Spider Diagrams}

Once the 6 characterization vectors are obtained, each island can be described through their values. In order to have a fast and graphical view of each island characteristics in one place, spider diagrams are constructed.

The spider diagrams obtained for the three lighthouse islands and the four follower islands are shown below (Figures 1-7).

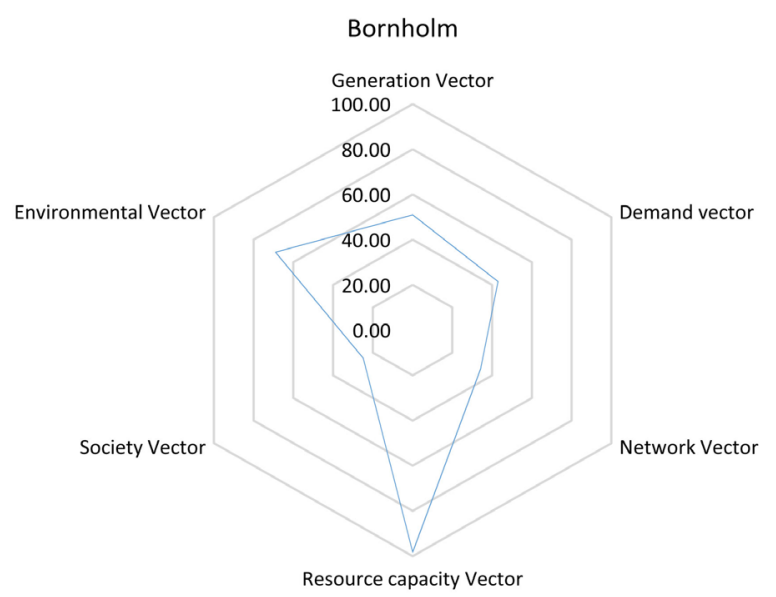

Figure 1. Bornholm spider diagram.

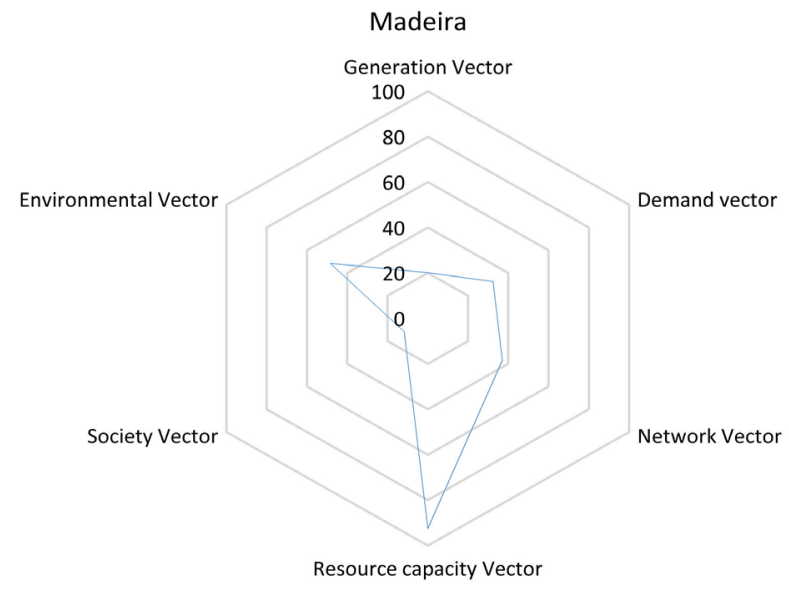

Figure 2. Madeira spider diagram. 


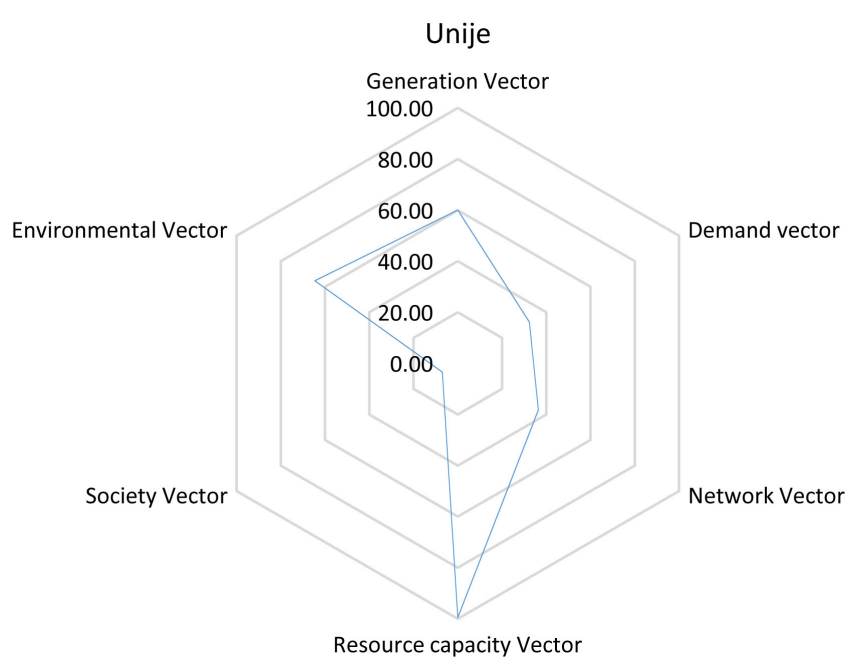

Figure 3. Unije spider diagram.

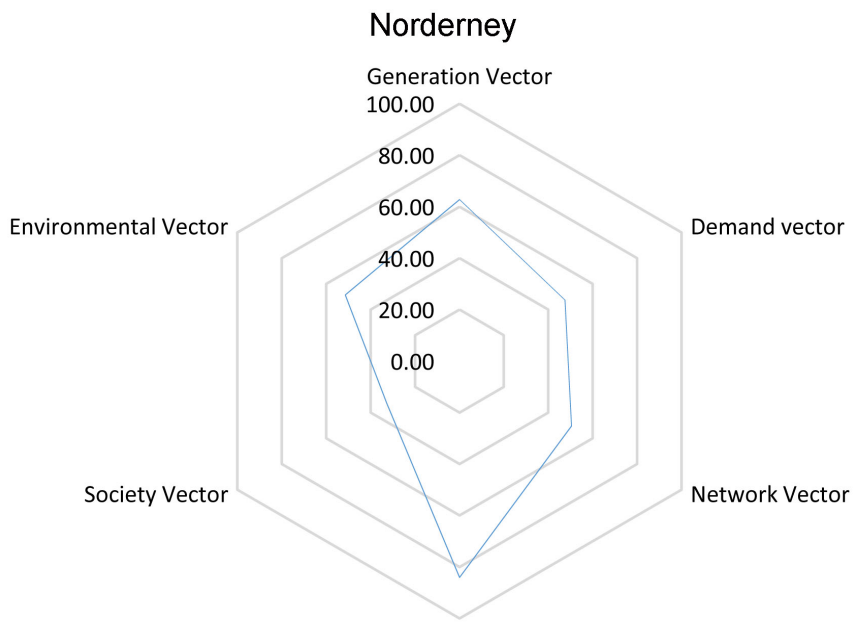

Resource capacity Vector

Figure 4. Norderney spider diagram.

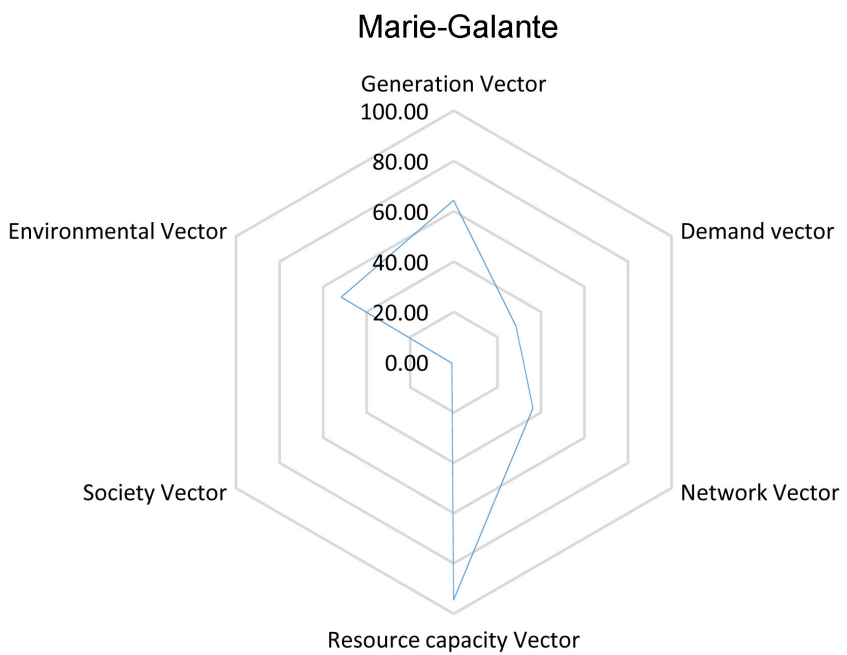

Figure 5. Marie-Galante spider diagram. 


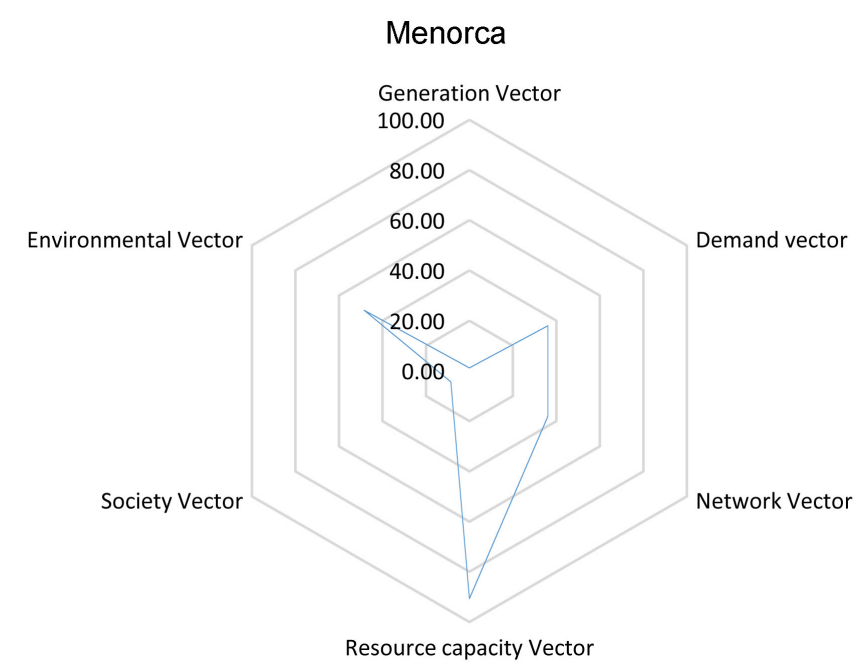

Figure 6. Menorca spider diagram.

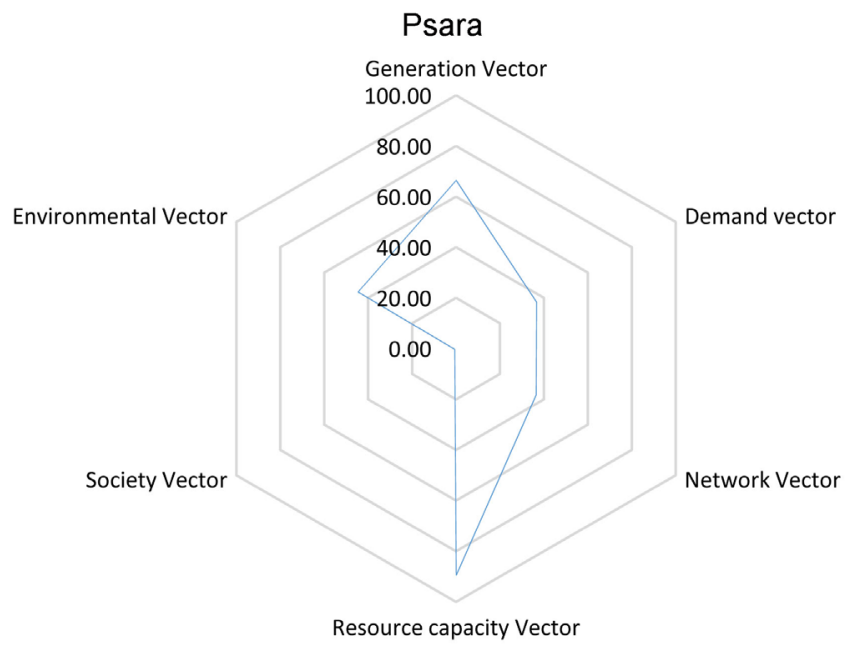

Figure 7. Psara spider diagram.

\section{EU Islands Data Gathering for Parameters}

The scope of this section is to explain the methodology followed for gathering the data for the parameters associated with the Island Categorization.

The set of islands in the EU is very extensive, and therefore the goal was to obtain data from large sets, in which the value of a parameter was given for as many islands as possible. As a first step, all parameters selected in task 2.1 were investigated, in order to identify those parameters that were more common or feasible to obtain. The search was improved by filtering the most common or relevant parameters.

As the data was gathered, a smaller set of representative and relevant systems were obtained. This filtering was carried out in accordance with the amount of representative data found for an island, which finally reduced the EU stock of islands with enough data to approximately 100 .

For these 100 islands, a specific search by island was performed in order to 
complete the information for these islands.

It is important to highlight that all information used to filter the islands and for the subsequent analysis, has been obtained from sources of information and open access databases that are available on the web. Whenever possible, attempts have been made to use official sources of information, such as from local system operators, from energy agencies, etc.

The list of islands and data was later enlarged with other contributions from task partners:

- Lighthouse and follower islands specific information for KPIs;

- Navigant-E3M_Islands and Energy in the EU Energy System final report [6];

- Mapping of islands' needs and resources in Croatia [4];

For certain parameters like desalinization data [9], wind speed [10], HDD [11], CDD [11], hydric stress [12], or GHG emissions [13] values, among others, were obtained from maps and the value for each island was obtained from its geographical position. Others, like Gross Domestic Product, GDP [8], Tourism data [14], Electricity and gas services [15] or Unemployment rate at regional level in EU [16], among others, were obtained from public EU sources that gather this information.

The final data set considered for the EU stock of islands contains 285 islands.

From them, not all the information was complete and therefore it was not possible to obtain all the KPIs for the whole EU stock of islands considered. As the information found for those islands was important in order to create representative reference islands, it was decided that those KPIs that were not complete would be completed with the average value from the set of islands for which that KPI value was available. In this way all the values needed for the vectors' composition of all the islands in the EU stock of islands considered, were obtained.

In order to store all data gathered and provide a starting template for KPI calculation an excel file was built. For each island the parameters gathered were included as a table in which columns were grouped by the vector for which the parameter was first used for.

\section{Reference Islands Identification and Value Analysis}

Once the information from the whole EU stock of islands was gathered and the characterization vectors defined, the set of data was analyzed through an algorithm based in the $k$-means quantization technique, obtaining seven reference islands that act as a representative view of the EU islands.

$K$-means [7] is an unsupervised classification (clustering) algorithm that groups objects into k groups based on their characteristics. The grouping is done by minimizing the sum of distances between each object and the centroid of its group or cluster. The quadratic distance is usually used. The analysis has been performed using Java Machine Learning Library [17].

The algorithm consists of three steps:

1) Initialization: once the number of groups, $k$, has been chosen, $k$ centroids are established in the data space, for example, by choosing them at random. 
2) Assignment of objects to centroids: each object in the data is assigned to its nearest centroid.

3) Maintain centroids: the position of the centroid of each group is maintained taking as the new centroid the position of the average of the objects belonging to that group.

Steps 2 and 3 are repeated until the centroids do not move or move below a threshold distance at each step.

The $k$-means algorithm solves an optimization problem, being the function to optimize (minimize) the sum of the quadratic distances of each object to the centroid of its cluster.

The objects, in our case the islands, are represented with real vectors of $d$ dimensions $\left(x_{1}, x_{2}, \cdots, x_{n}\right)$ and the $k$-means algorithm constructs $k$ groups where the sum of distances of the objects is minimized, within each group $S=\left\{S_{1}, S_{2}, \cdots, S_{k}\right\}$ to its centroid. The problem can be formulated as follows:

$$
\min _{S} E\left(\mu_{i}\right)=\min _{S} \sum_{i=1}^{k} \sum_{x_{j} \in S_{i}}\left\|x_{j}-\mu_{i}\right\|^{2}
$$

where $S$ is the data set whose elements are the $x_{j}$ objects represented by vectors, where each of its elements represents a characteristic or attribute. We will have $k$ groups or clusters with their corresponding centroid $\mu_{i}$.

In each centroid update, from the mathematical point of view, we impose the necessary end condition to the function $E\left(\mu_{i}\right)$ which, for the previous formula is:

$$
\frac{\partial E}{\partial \mu_{i}}=0 \rightarrow \mu_{i}^{(t+i)}=\frac{1}{\left|S_{i}^{(t)}\right|} \sum_{x_{j} \in S_{i}^{(t)}} x_{j}
$$

And the average of the elements in each group is taken as the new centroid. The final centroids for each cluster after the execution of the algorithm are taken as the reference islands.

The main advantages of the $k$-means method are that it is a simple and fast method. But it is necessary to choose the $k$-value and the result depends on the initialization of the centroids. In principle it does not converge to the global minimum but to a local minimum. In order to find the global optimum, and since the complexity of the problem at hand allows a fast execution of the algorithm, it has been run several times in order to explore the total space of solutions.

In order to find the number of clusters of the $k$-means algorithm we use the "Elbow method" [18]. Once the values of the function $E\left(\mu_{i}\right)$ are obtained after applying the $k$-means from 1 to n clusters, a linear graph of $E\left(\mu_{i}\right)$ with respect to the number of clusters is represented. In this graph we should appreciate a sudden change in the evolution of the function, with the line represented having a shape similar to an arm and its elbow. The point at which this sudden change is observed will tell us the optimum number of clusters to be selected for that data set; or to put it another way: the point representing the elbow of the arm will be the optimum number of clusters for that data set (Figure 8). 


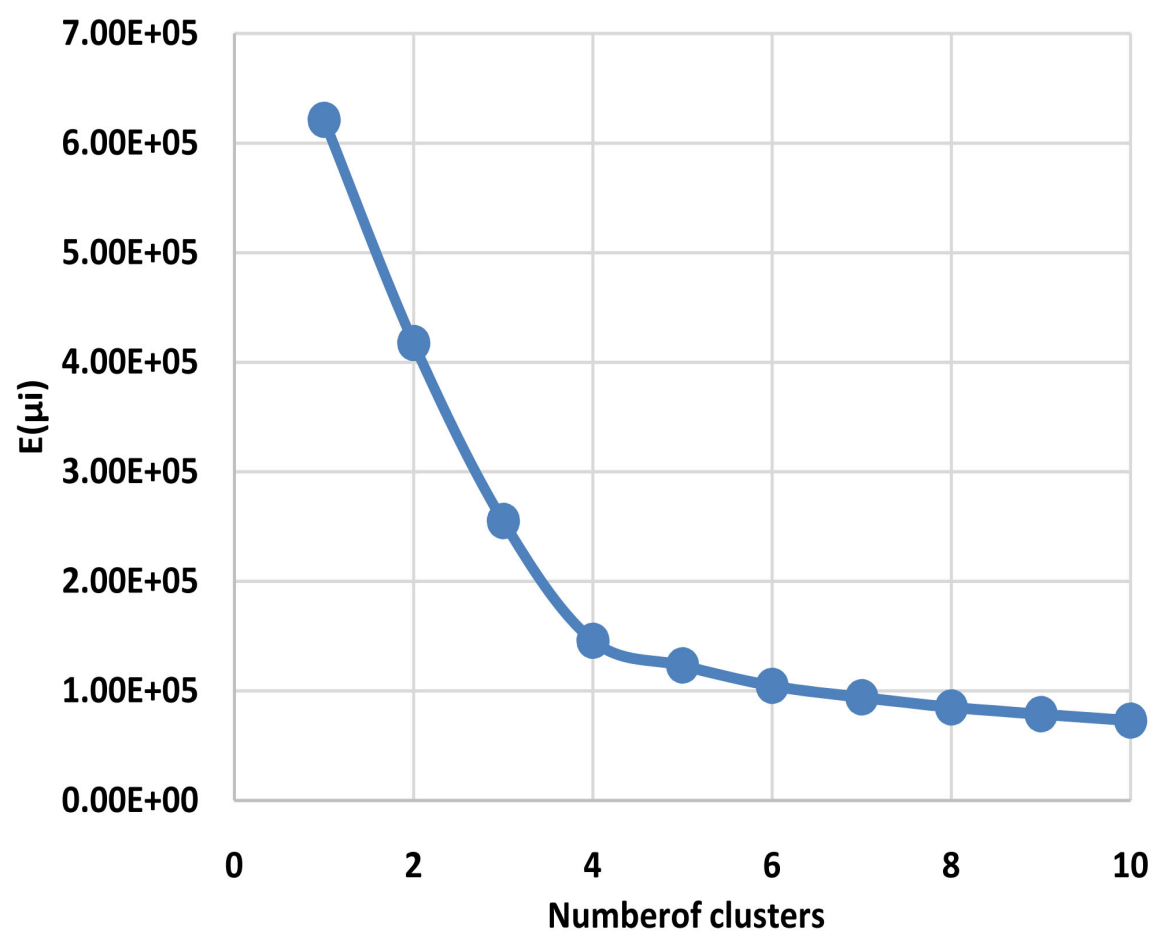

Figure 8. Variation of $\mathrm{E}(\mu \mathrm{i})$ vs Number of clusters.

As seen in the graph the sudden change is obtained at $k=4$, nevertheless, to obtain a wider number of clusters and more granularity in the classification we opt to choose a higher number of clusters, therefore $k=7$.

The Reference islands obtained are shown.

\subsection{Construction of Reference Islands and Their Vectors}

The Reference islands obtained have the following values for the characterization vectors defined in Table 1 .

This information can also be shown through the spider diagrams of the seven Reference Islands in Figure 9.

Table 1. Obtained Reference islands and their values for characterization vectors.

\begin{tabular}{ccccccc}
\hline \multirow{2}{*}{$\begin{array}{c}\text { Reference } \\
\text { Island }\end{array}$} & \multicolumn{5}{c}{ Characterization vectors } \\
\cline { 2 - 7 } & Generation & Demand & Network & Resource Capacity & Society & Environmental \\
\hline Type 1 & 15.28 & 35.82 & 36.45 & 90.47 & 2.33 & 43.65 \\
Type 2 & 2.73 & 36.48 & 36.42 & 94.34 & 1.75 & 42.88 \\
Type 3 & 12.42 & 41.03 & 36.81 & 95.39 & 27.66 & 44.36 \\
Type 4 & 2.92 & 47.42 & 36.13 & 90.95 & 24.97 & 71.51 \\
Type 5 & 8.94 & 35.25 & 35.32 & 89.88 & 4.66 & 22.56 \\
Type 6 & 55.30 & 46.34 & 36.52 & 97.01 & 21.96 & 68.17 \\
Type 7 & 59.02 & 51.06 & 36.67 & 4.36 & 27.87 & 53.94
\end{tabular}



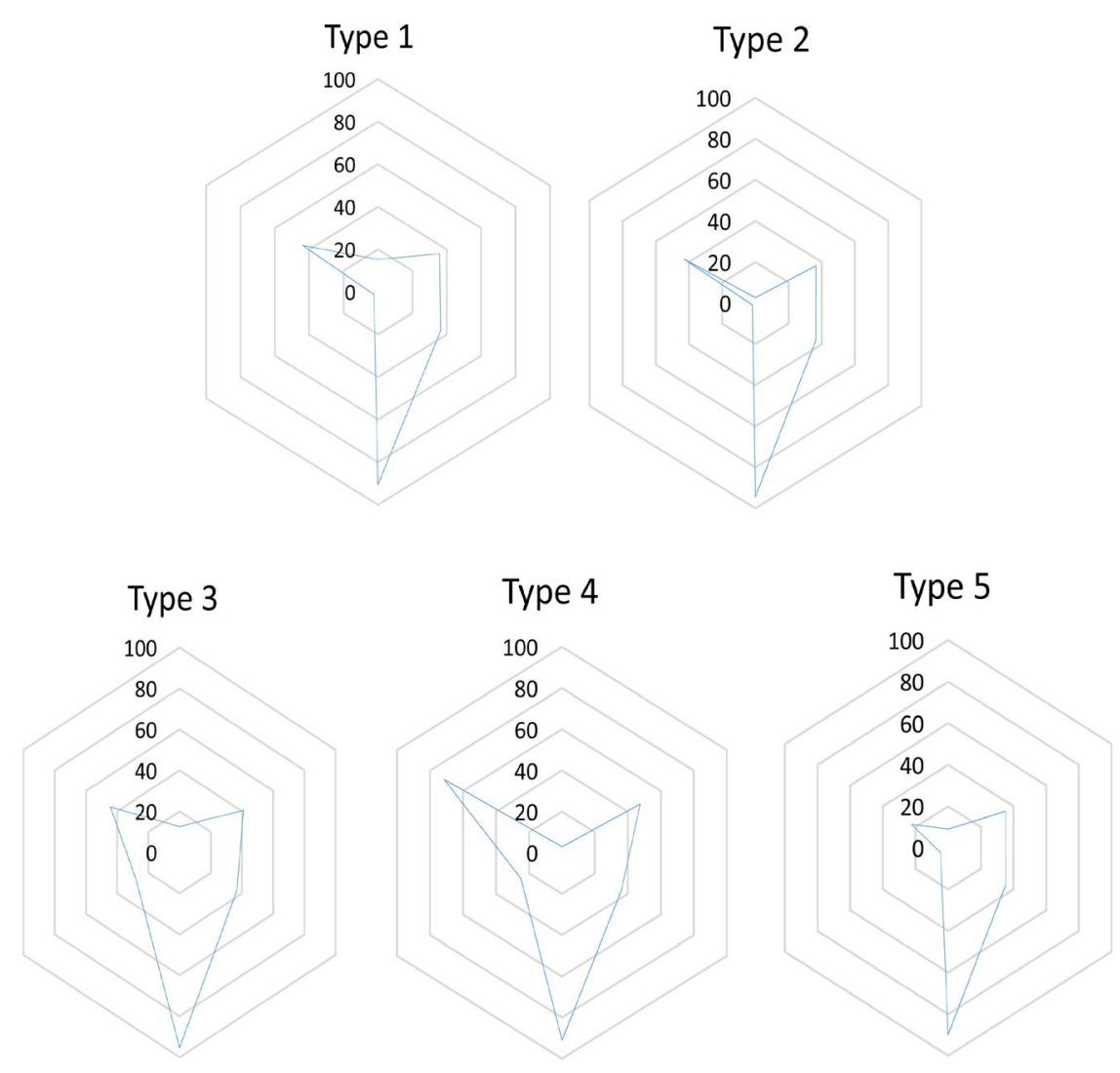

Type 6

Type 7

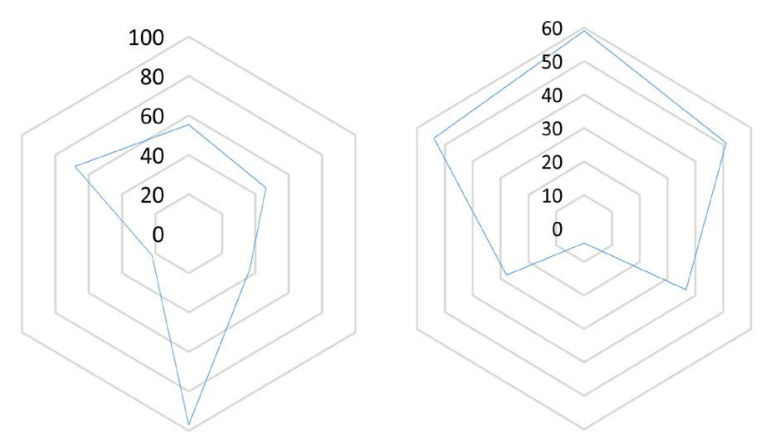

Figure 9. Reference islands spider diagrams.

\subsection{Representativeness of the Reference Islands}

Through the different vectors it is possible to provide a description of the meaning of each spider diagram for each reference island, which certainly will help to better understand their representativeness.

\section{Type 1 Reference island}

Type 1 Reference island has a low level of renewable generation. The energy demand by population, considering electricity, vehicles and heating and cooling devices is under the EU average. The network has a medium level of contingencies or capacity to damp hazardous situations. The island still has the capacity to implement new renewable generation. Society value indicates low GDP per ca- 
pita or high unemployment rates, meanwhile environmental issues are within the EU average.

Type 2 Reference island

Type 2 Reference island has only conventional generation without interconnection. The energy demand by population, considering electricity, vehicles and heating and cooling devices is under the EU average. The network has a medium level of contingencies or capacity to damp hazardous situations. The island has the capacity to implement new renewable generation to cover its demand. Society value indicates low GDP per capita or high unemployment rates, meanwhile environmental issues are within the EU average.

Type 3 Reference island

Type 3 Reference island has only conventional generation without interconnection. The energy demand by population, considering electricity, vehicles and heating and cooling devices is under the EU average. The network has a medium level of contingencies or capacity to damp hazardous situations. The island has the capacity to implement new renewable generation to cover its demand. Society value indicates high GDP per capita or low unemployment rates, and environmental issues are within the $\mathrm{EU}$ average.

\section{Type 4 Reference island}

Type 4 Reference island has only conventional generation without interconnection. The energy demand by population, considering electricity, vehicles and heating and cooling devices is under the EU average. The network has a medium level of contingencies or capacity to damp hazardous situations. The island has the capacity to implement new renewable generation to cover its demand. Society value indicates high GDP per capita or low unemployment rates, and environmental issues are well positioned with respect to the EU average.

\section{Type 5 Reference island}

Type 5 Reference island has a low level of renewable generation. The energy demand by population, considering electricity, vehicles and heating and cooling devices is under the EU average. The network has a medium level of contingencies or capacity to damp hazardous situations. The island has the capacity to implement new renewable generation to cover its demand. Society value indicates low GDP per capita and high unemployment rates, and environmental issues are positioned below the EU average.

\section{Type 6 Reference island}

Type 6 Reference island has high levels of renewable generation and manageable renewable generation. The energy demand by population, considering electricity, vehicles and heating and cooling devices is above the EU average. The network has a medium level of contingencies or capacity to damp hazardous situations. The island has the capacity to implement new renewable generation to cover its demand. Society value indicates GDP per capita and employment rates are above the EU average, and environmental issues are well positioned with respect to the EU average. 


\section{Type 7 Reference island}

Type 7 Reference island has high levels of renewable generation and manageable renewable generation and with interconnection to export any additional energy generation. The energy demand by population, considering electricity, vehicles and heating and cooling devices is above the EU average. The network has a low level of contingencies and capacity to damp hazardous situations. The island has already covered its demand with renewable sources and therefore there is no need for further investment in new renewable generation to cover its demand. Society value indicates GDP per capita and employment rates are above the EU average, and environmental issues are well positioned with respect to the EU average.

\subsection{Linkage of Project Islands with Reference Islands}

In the same way that any new island would be characterized and linked with one of the Reference islands obtained, the project islands have also been linked with the Reference islands.

The linkage is done through a mathematical methodology, using the values of the vectors of the reference islands, and calculating the distance from the specific island to the Reference islands. Or directly through a comparison of the spider diagram obtained for the specific island with those from the Reference islands. This linkage helps to identify those solutions proposed in the project that could be later exported or give ideas for the Investment Plan of any new island linked with the same typology of Reference Island.

Following, the linkages that were found with the project islands are shown in Figures 10-12.

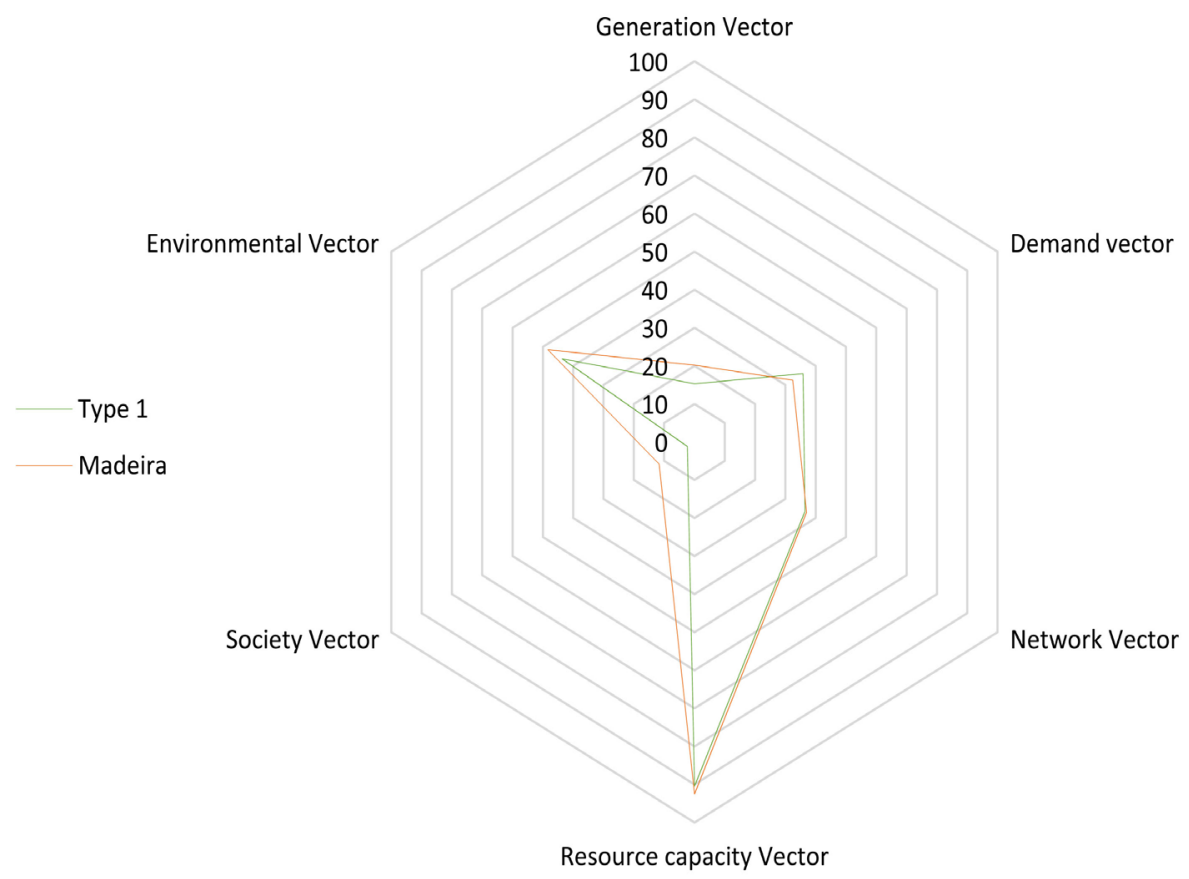

Figure 10. Type 1 and Madeira spider diagrams. 


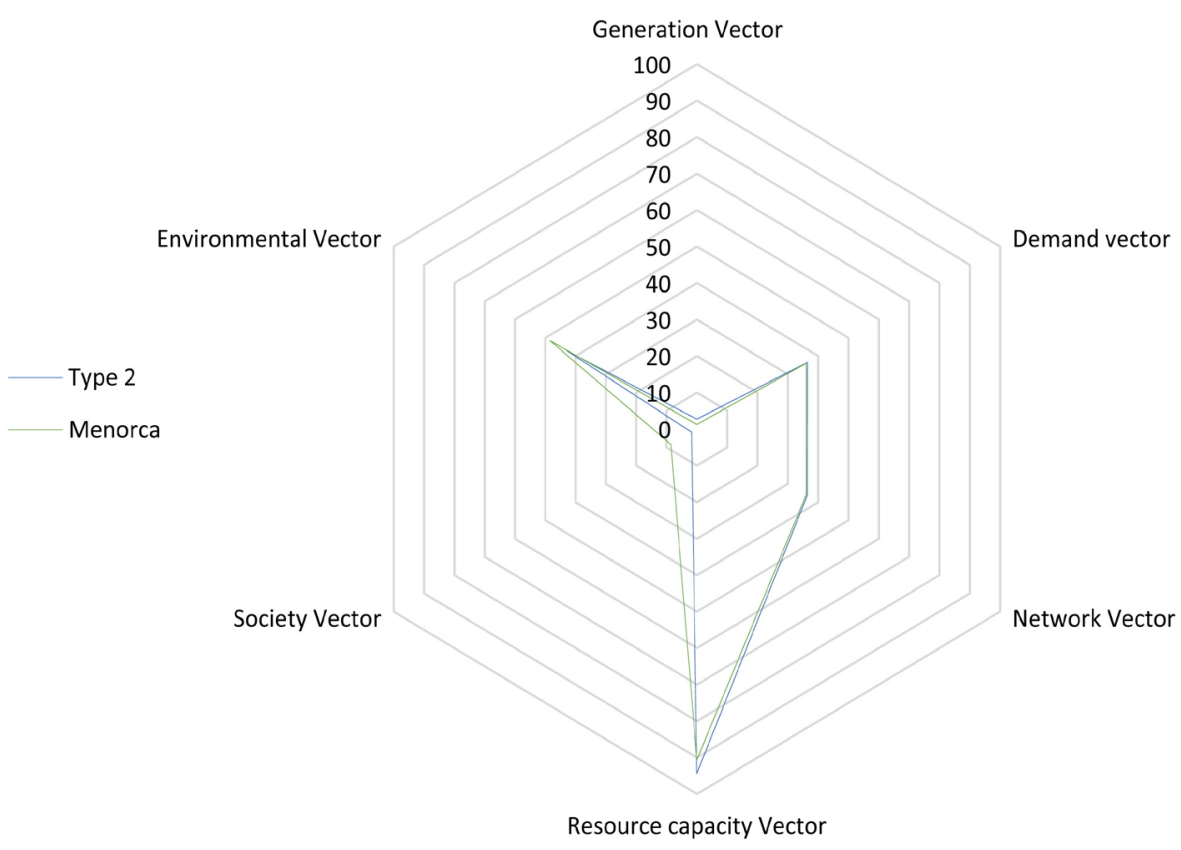

Figure 11. Type 2 and Menorca spider diagrams.

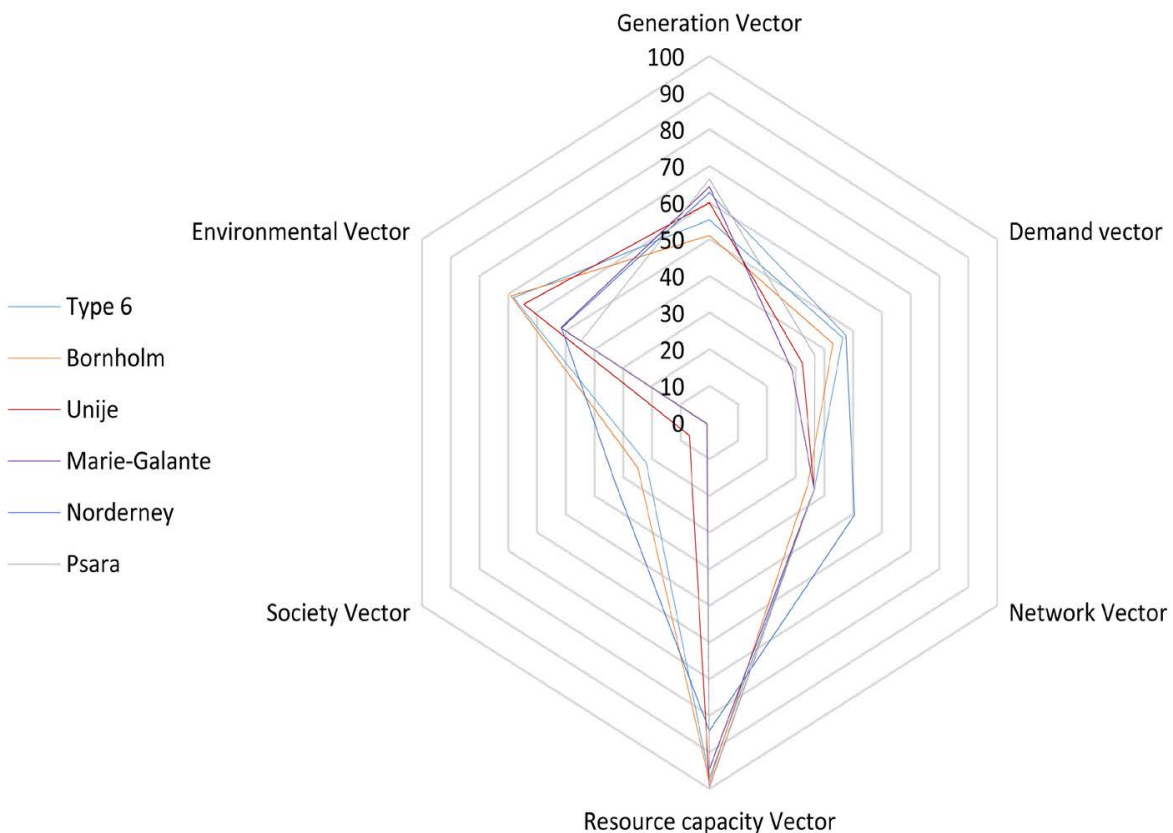

Figure 12. Type 6, Bornholm, Unije, Marie-Galante, Norderney and Psara spider diagrams.

The INSULAE project methodology of linking any new island to a reference island is additive. It leads to a growing list of recommendations and examples of solutions that can be applied to any follower island.

For example, Norderney, Marie-Galante and Psara islands have a linkage with Reference island type 6 as well as Bornholm and Unije, and therefore for their Investment Plan examples on the challenges faced and solutions implemented in Bornholm and Unije could be shared, after implementation they then become 
real life examples of interventions on Reference island type 6.

On the other hand, Menorca island has been identified with Reference island type 2 where there is no Lighthouse island identified. In this case, Type 1 Reference island has a close relationship with type 2 reference island if the generation vector is ignored, and therefore problems and solutions from Madeira could be shared in order to prepare its Investment Plan.

Just like Menorca island, there will be more islands identified with Reference islands for which the project INSULAE has not been linked to. The solution is that vector characterization enables us to look at one or two of the vectors that are considered of special relevance for the island and search for any of the project islands with similar values for that vector or group of vectors. Therefore, information from the solutions related to specific vectors are good possibilities for the Investment plan of an island with similar vector values. Alternatively, by using the Artelys tool and the enriched library of smart grid systems and interventions that will be identified through WP4, a mix of possible interventions that are characterized with the adequate technical, environmental, economic, and social metrics could be made available to the IPT end-user for the island under consideration. These interventions will provide input to the calculation engine, and create synergies resulting from complex multi-network interactions. This will help define the needs of the Reference islands identified, as an initial grouping of main characteristics, and the particular needs of each island that will utilize the IPT tool.

\section{Conclusions}

Overall objective of INSULAE project, to aid decarbonization of islands, should be of interest to any isolated electrical system, and especially to EU islands that have similarities with the INSULAE project islands. In order to improve the replicability of solutions in other islands with their own characteristics and very diverse conditions, the presented characterization methodology helps new islands to match their problems with similar situations and possible solutions. Then they will construct their own investment plans with confidence that their decarbonization and development goals will be achieved.

The planned INSULAE Investment Planning Tool (IPT), that will guide EU island decision makers in the design of cost-effective Action Plans for decarbonizing their energy systems, will integrate the composition of the characterization vectors, and show the spider diagram obtained. This tool will also provide examples of the solutions implemented in lighthouse or follower islands matched through the typology of Reference island.

It has been seen that the project islands do not cover all the Reference typologies obtained, and that solutions implemented in INSULAE project will not cover all the problems that could be found among EU Islands. From here a future goal can be established. Integrate the Reference islands methodology and through the BRIDGE initiative, implement new solutions in islands that could help cover the complete set of Reference islands. 


\section{Acknowledgements}

The authors would like to express their appreciation to INSULAE partners, with special focus on the Islands of Bornholm, Unije, Madeira, Marie-Galante, Menorca, Norderney and Psara for the detailed data provided and to University of Zagreb (UZ-FSB), RINA Consulting SPA, ARTELYS SA, Diktyo AeiforikonNison Toy Aigaiou AE (DAFNI), Danmarks Tekniske Universitet (DTU), WWF Adria and DNV GL Netherlands B.V. for contributing with valuable comments under the INSULAE project framework.

The research leading to these results is part of the work carried out in the framework of INSULAE-Maximizing the impact of innovative energy approaches in the EU islands' project. This project has received funding from the European Union Horizon 2020 research and innovation Programme under grant agreement No. 824433.

\section{Conflicts of Interest}

The authors declare no conflicts of interest regarding the publication of this paper.

\section{References}

[1] European Commission (2018) Decarbonising Energy Systems of Geographical Islands. https://cordis.europa.eu/programme/id/H2020_LC-SC3-ES-4-2018-2020

[2] United Nations (2020) Sustainable Development Goals. https://www.un.org/sustainabledevelopment/

[3] de Urtasun, L.G., Vicente, S.B. and Hernández, N.G. (2016) Smart Grid Functionalities Evaluation. Smart Grid and Renewable Energy, 7, 175-189. https://doi.org/10.4236/sgre.2016.76014

[4] Mimica, M. and Krajačić, G. (2019) Advanced Renew Islands Method with Quantitative Mapping of Islands' Needs and Resources. Digital Proceedings of the 14th Conference on Sustainable Development of Energy, Water and Environment Systems-SDEWES, Dubrovnik, 1-6 October 2019.

[5] Smart Island Energy Systems, SMILE Project. Deliverable D6.1 (2018) Report on Selected Evaluation Indicators. https://www.h2020smile.eu/press-downloads/

[6] Islands and Energy Islands in the EU Energy System. https://asset-ec.eu/wp-content/uploads/2018/04/ASSET_4_EnergyIsland.pdf

[7] University of Oviedo. K-Means Algorithm. https://www.unioviedo.es/compnum/laboratorios_py/kmeans/kmeans.html\#kmeans

[8] GDP per Capita in EU Regions. https://en.wikipedia.org/wiki/List_of_European_regions_by_GDP

[9] Desalinization Data in EU (2011). http://www.imida.es/documents/13436/877249/06_REPORT_ON_WATER+DESA LINATION.pdf/ae6203f4-68f9-4a37-b728-22b5d247909e

[10] Wind Speed. https://globalwindatlas.info/

[11] HDD \& CDD Data. https://www.eurima.org/uploads/ModuleXtender/Publications/13/EURIMA-ECOF YS_VII_report_p66-104_ANNEXES1.pdf 
[12] AQUEDUCT (2019) Hydric Stress Data.

https://www.wri.org/applications/aqueduct/water-risk-atlas/\#/?advanced=false\&bas emap=hydro\&indicator=bws_cat\&lat $=41.74115167734524 \& \operatorname{lng}=27.3781489580869$ $7 \&$ mapMode $=$ view\&month $=1 \&$ opacity $=0.5 \&$ pondera-tion $=$ DEF\&predefined $=$ false \&projection $=$ absolute $\&$ scenario $=$ optimistic $\&$ scope $=$ baseline $\&$ timeScale $=$ annual $\& y e$ ar=baseline\&zoom $=6$

[13] IMGUR (2016) GHG Emission Data. https://imgur.com/r/europe/cEmjl

[14] Eurostat (2018) Tourism Data in EU Regions.

https://ec.europa.eu/eurostat/statisticsexplained/index.php?title=Tourism_statistics _at_regional_level\#Number_of_overnight_stays

[15] Council of European Energy Regulators (2016) Benchmarking on Electricity and Gas Service Quality and Reliability.

https://www.ceer.eu/documents/104400/-/-/963153e6-2f42-78eb-22a4-06f1552dd34c

[16] Eurostat (2019) Unemployment Rate at Regional Level in EU.

https://ec.europa.eu/eurostat/statistics-explained/index.php/Unemployment_statisti cs_at_regional_level

[17] Abeel, T., de Peer, Y.V. and Saeys, Y. (2009) Java-ML: A Machine Learning Library. Journal of Machine Learning Research, 10, 931-934.

[18] Kodinariya, T.M. and Makwana, P.R. (2013) Review on Determining Number of Cluster in $K$-Means Clustering. International Journal of Advance Research in Computer Science and Management Studies, 1, 90-95. 